\title{
2nd Workshop on Patent Text Mining and Semantic Technologies (PatentSemTech2021)
}

\author{
Ralf Krestel \\ ralf.krestel@hpi.de \\ Hasso Plattner Institute \\ University of Potsdam, Germany \\ Florina Piroi \\ florina.piroi@researchstudio.at \\ Data Science Studio \\ Vienna, Austria
}

\author{
Hidir Aras \\ hidir.aras@fiz-karlsruhe.de \\ FIZ Karlsruhe \\ Eggenstein-Leopoldshafen, Germany \\ Allan Hanbury \\ allan.hanbury@tuwien.ac.at \\ TU Wien, Austria
}

\author{
Linda Andersson \\ linda.andersson@artificialresearcher.com \\ Artificial Researcher IT GmbH \\ Vienna, Austria \\ Dean Alderucci \\ dalderuc@cs.cmu.edu \\ Carnegie Mellon University, \\ Center for AI and Patent Analysis \\ Pittsburgh, USA
}

\begin{abstract}
Information retrieval plays a crucial role in the patent domain. With the success of deep learning (DL) in other domains, patent practitioners and researchers are increasingly developing DL-based approaches to support experts in the patenting process or to automate processes for patent analysis. AI-enhanced information retrieval systems can improve patent search but also require lots of annotated data. When working with patent data, particular challenges arise that call for adaption and novel approaches of general IR and AI methods. with this workshop series we want to establish a two-way communication channel between industry and academia from relevant fields in information retrieval, such as natural language processing (NLP), text and data mining (TDM), and semantic technologies (ST), in order to explore and transfer new knowledge, methods and technologies for the benefit of industrial applications as well as support interdisciplinary research in applied sciences for the intellectual property (IP) and neighbouring domains.
\end{abstract}

\section{CCS CONCEPTS}

- Information systems $\rightarrow$ Information retrieval; Digital libraries and archives; Data mining; • Applied computing $\rightarrow$ Document management and text processing; • Computing methodologies $\rightarrow$ Natural language processing.

\section{KEYWORDS}

patent analysis, text mining, semantic technology, deep learning

\section{ACM Reference Format:}

Ralf Krestel, Hidir Aras, Linda Andersson, Florina Piroi, Allan Hanbury, and Dean Alderucci. 2021. 2nd Workshop on Patent Text Mining and Semantic Technologies (PatentSemTech2021). In Proceedings of the 44th International ACM SIGIR Conference on Research and Development in Information Retrieval (SIGIR '21), July 11-15, 2021, Virtual Event, Canada. ACM, New York, NY, USA, 4 pages. https://doi.org/10.1145/3404835.3462816

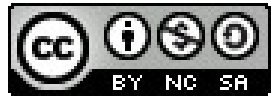

This work is licensed under a Creative Commons Attribution-NonCommercialShareAlike International 4.0 License.

SIGIR '21, July 11-15, 2021, Virtual Event, Canada.

(C) 2021 Copyright held by the owner/author(s).

ACM ISBN 978-1-4503-8037-9/21/07.

https://doi.org/10.1145/3404835.3462816

\section{MOTIVATION}

The PatentSemTech2021 workshop is the second in a series of workshops that has started in 2019, in conjunction with the SEMANTiCS 2019 conference in Karlsruhe [1]. The aim of the workshop series is to establish a long-term collaboration and a two-way communication channel between industry and academia from relevant fields in information retrieval and related areas such as natural language processing (NLP), text and data mining (TDM), and semantic technologies (ST), in order to explore and transfer new knowledge, methods and technologies for the benefit of industrial applications as well as support interdisciplinary research in applied sciences for the intellectual property (IP) and neighbouring domains, such as scientific text analysis from bio-medicine, chemistry, etc.

Challenges of using IP data for IR.. From the definition of a search task perspective, users of patent information systems are highly specialized information professionals, who cooperate with research and/or legal departments in their institutions or companies. Finding the right information is business critical in the patent domain. There are high requirements for the correctness and completeness of the data to search through, for the efficiency of the search interface, for the trustworthiness of the provider, and for the quality of the search results. For general natural language documents (such as news articles, or Wikipedia articles) there are a variety of tools and methods to process and prepare them for a specific task. It is a most challenging undertaking to adapt or re-design such tools to address the requirements of working with patent and legal documents.

Patent Data Traits. Patents are a type of scientific text which is complex and difficult to analyse compared to common texts. Some reasons are:

- Patents, as a corpus and as a single document, are both very heterogeneous. A patent corpus covers very diverse scientific subject areas, such as chemistry, pharmacology, mining, and all areas of engineering, with the consequence that all kinds of terminology can be found in a patent corpus.

- A patent corpus usually covers a long time span, often from the 1950 s to the present.

- Typographical errors are not uncommon, since many patents in their machine-readable form are derived from OCR-processing and machine-translation. 
- Patents are composed of detailed descriptions of the invention and the claims. As a result, patents are on average two to five times longer than scientific articles.

- Patents are usually characterized by the use of legal language to describe what is claimed by the invention, making them hard to understand for a layperson.

Why work with Patent Data? Patent data, besides its challenging aspects, comes with a richness of facets that makes it interesting for text-mining and semantic methods:

- It constitutes a huge corpus of scientific-technical documents for a variety of technological domains.

- It is rich in available meta-data such as spatial data, bibliographic data, classifications, temporal data, etc.

- Patents describe essential scientific-technical knowledge enclosing solutions for real-world applications.

- Patent data is complementary knowledge to scientific literature, e.g., it describes chemical and physical properties or bio-science knowledge for drug-target-interaction, which appears first in patents and is mostly not published elsewhere.

Suitability to SIGIR.. Given the traditional connection of patent retrieval and SIGIR $[5,6]$ on the one hand, and the prevalent use of deep learning (DL) methods in information retrieval [7] on the other hand, a dedicated workshop with a focus on deep learning methods for patent retrieval and analysis would help to bring state-of-theart IR methods into the patent domain. Further, patent analysis and retrieval are inherently application-driven and IR research is highly relevant for IP practitioners. Within SIGIR, we find the ideal setting to discuss and make connections between IR researchers and practitioners at the intersection of deep learning and patent retrieval. IP practitioners will benefit from the IR community by learning novel IR methods that could possibly be applied to patentrelated texts, and IR researchers learn about publicly available, wellannotated, large document collections and exciting tasks suitable for IR research.

\section{MAIN TOPICS}

We encourage submission of new methods for scientific text and patent analysis which can be applied, e.g., for empowering new forms of search and retrieval as well as supporting large-scale technology analysis for the IP and neighboring domains. Our aim is to motivate researchers in academia and industry to explore, among others,

- text and data mining methods, in particular deep learning based methods,

- semantic enrichment of large amounts of scientific texts, e.g., to aid retrieval systems or generate training data,

- exploit scientific-technical information outside of the IP world, for example, by interlinking valuable knowledge sources from domain-specific knowledge graphs (bio-pharma, chemistry, engineering, etc.) or the Linked Open Data (LOD) cloud.

Further, we welcome papers along our main theme: Application of Deep Learning Methods for Patent Retrieval and Analysis, that, among others,

- present novel datasets for training deep learning models,
- explore IP applications with underlying advanced NLP, TDM and artificial intelligence methods, e.g., applying and adapting DL methods for various domain-specific tasks,

- apply enhanced machine learning and semantic technologies to enrich and analyse patent texts, e.g., in order to contribute to use cases such as technology analysis, trend analysis, semantic patent landscaping, competitor analysis, etc.

- show proof-of-concept patent and technology analysis use cases, such as patent landscaping, portfolio analysis, white and hotspot analysis, technology trends analysis, etc.

- evaluate new visual user interface concepts for exploring and analysing large datasets of scientific texts.

Current research trends in the patent domain show interesting developments in all of these areas. Novel resources area presented, e.g., patent-specific word embeddings [9] and novel tasks for automation [8]. Efforts in patent landscaping ${ }^{1}$ and visualization are also increasing. ${ }^{2} \mathrm{~A}$ literature review resource with the aim to bridge over different scientific text genres is developed by Artificial Researcher IT $\mathrm{GmbH},{ }^{3}$ based upon research presented in [2].

\section{PROGRAM}

The program will reflect the main workshop series theme: bridging the gap between patent practitioners and IR researchers by inclusion of two keynote speakers, one from the patent industry and one from IR research. Further, the full day workshop will feature selected talks from industry and academia on various topics associated with text mining, machine learning and semantic technologies applied to patents or other related domain-specific texts. We will also try to engage national and international patent office (such as WIPO and EPO) and active companies in industry (such as Patsnap, Octimine, Google Patents, InfoChem, etc.) and initiatives like "The Lens" to contribute to the workshop as a partner, either with scientific contributions or as panel members.

Panel Discussion. In the Intellectual Property community, it has been established that some technology know-how is only obtainable via patents. Depending on the technical fields, the information uniquely discovered in patents ranges from $56 \%$ to $80 \%$ [3]. It is therefore crucial to have powerful IR systems in place that more and more incorporate AI methods. In the panel discussion we would like to emphasise patents as a resource for different text mining solutions, such as terminology extraction, part of literature review tools, or a source for extracting problem-solving information [4]. Information extracted from patents can contribute to the scientific communities with new know-how as well as identifying emerging technologies for planning future strategy research policies, such as in applied research related to COVID-19 pandemic ${ }^{4}$. With the theme of the panel discussion being Artificial Intelligence and Patent Analysis: Friends or Foes? we hope to shed light onto various aspects related to practical and theoretical issues of automation in the patent domain.

\footnotetext{
${ }^{1}$ https://github.com/google/patents-public-data

${ }^{2}$ https://wipopearl.wipo.int/en/conceptmap

${ }^{3}$ https://passageretrieval.artificialresearcher.com/

${ }^{4}$ https://www.fiz-karlsruhe.de/nachricht/fiz-special-corona-applying-semantic-

technologies-knowledge-discovery-patents-case-study
} 
Program Committee. Our program committee consists of experts from the IP industry and academia.

- Siegfried Handschuh, University of St. Gallen, Switzerland

- Christoph Hewel, Patent Attorney, Germany

- Julian Risch, deepset GmbH, Germany

- Florian Matthes, TU Munich, Germany

- Rene Hackl-Sommer, FIZ Karlsruhe, Germany

- Anthony Trippe, Patinformatics, Ireland

- Sam Arts, KU Leuven

- Paul Groth, University of Amsterdam, Netherlands

- Hans-Peter Zorn, inovex Gmbh, Karlsruhe, Germany

- Michael Natterer, Dennemeyer Octimine GmbH, Germany

\section{CONTRIBUTIONS}

The scientific submissions will be screened by the workshop organizers, then enter a double-blind reviewing process. Workshop post-proceedings will be published as Open Access on TU Wien's reposiTUm infrastructure ${ }^{5}$ and if possible as CEUR workshop proceedings. ${ }^{6}$ Selected papers will be invited to submit their paper to Elsevier's World Patent Information (WPI) journal ${ }^{7}$ for inclusion in the virtual special issue on "Patent Text Mining and Semantic Technologies".

Types of Contributions. We welcome full research papers that describe original work. The following topics are of particular interest.

- Novel applications of State of the Art in the IP domain

- Applications for novel tasks in the IP domain

- Novel user interfaces for the IP domain

- Novel evaluation or analysis insights in the IP domain

- Novel benchmark datasets

- Surveys/Overview related to the IP domain

Given the importance of training data for machine learning research, we especially emphasize the importance of describing the utilized resources in-depth and encourage to make the datasets publicly available if possible.

Case Studies, Demos, and Resource Papers. We further welcome short papers in the form of demonstration, case study, or resource papers. Case study papers should describe a focused case study making use of semantic technologies or machine learning, an interesting IP-related task descriptions or best practices for patent analysis. Demo papers should describe in-use systems or prototype implementation of semantic technologies or deep learning approaches that can be presented and demonstrated. The focus of the demo can be on processing or analysing data from the IP domain, or focused on user experience or interfaces. Resource papers should describe novel resources related to patents or related legal documents. Further, they can describe external resources to augment IP datasets, e.g., linked open data.

\footnotetext{
${ }^{5}$ https://repositum.tuwien.at/?locale=en

${ }^{6} \mathrm{http}: / /$ ceur-ws.org/

${ }^{7}$ https://www.journals.elsevier.com/world-patent-information

${ }^{8} \mathrm{https}$ ://www.journals.elsevier.com/world-patent-information/call-for-papers/textmining-and-semantic-technologies
}

\section{ORGANIZERS}

Dr. Ralf Krestel. is a senior researcher and head of the Web Science Group at Hasso Plattner Institute at University of Potsdam, Germany. His research centers around text mining, information retrieval, recommender systems, natural language processing, and machine learning. He has published multiple papers on patent retrieval, e.g., at the recommender systems conference.

Dr. Hidir Aras. is a senior researcher and project manager for text and data mining at FIZ Karlsruhe. His applied research interests include big data analytics, text and data mining, and semantic analysis of patent information. In his previous research at the University of Bremen, where he finished his doctoral thesis, he focused on systems that employ Semantic Web technologies, social networking paradigms and $\mathrm{HCI}$ for the interaction and exploration of large information spaces on the Web.

Dr. Allan Hanbury. is Professor for Data Intelligence and head of the E-Commerce Research Unit in the Faculty of Informatics, TU Wien, Austria. He is also faculty member of the Complexity Science Hub Vienna. He was scientific coordinator of the EU-funded Khresmoi Project on medical and health information search and analysis, and is co-founder of contextflow, the spin-off company commercialising the radiology image search technology developed in the Khresmoi project. He is coordinator of DoSSIER, a Marie Curie Innovative Training Network, educating 15 doctoral students on domainspecific systems for information extraction and retrieval. He is author or co-author of over 160 publications in refereed journals and refereed international conferences. Most recently, he was Tutorial Co-Chair of the European Conference on Information Retrieval (ECIR) 2020; Short Paper Co-Chair of the ECIR 2018; Programme Committee Co-Chair of the European Big Data Value Forum 2018, Vienna, Austria; Special Session Chair of the ACM International Conference on Multimedia Retrieval (ICMR) 2017; and Programme Committee co-chair for ECIR in 2015.

Ms. Linda Andersson. TU Wien alumni and entrepreneur, Ms. Linda Andersson is the CEO of the start-up Artificial Researcher-IT GmbH. In 2018 they launched the product idea 'Artificial Researcher in Science' which received the Commercial Viability Award from the Austrian Angel Investors Association. Ms Andersson has over 17 years of experience in the text mining industry and worked with different aspects of scientific literature text mining. Parallel to her academic studies, she has been working in the Intellectual Property industry, designing different domain-specific patent text mining solutions. Her PhD Thesis, "The Essence of Patent Text Mining", was a product of close collaboration with the Intellectual Property industry. Ms Andersson is currently the guest editor for the World Patent Information Issue associated with "Text Mining and Semantic Technologies in the Intellectual Property Domain".

Dr. Florina Piroi. is a senior researcher now at the Research Studios Austria, Data Science group, with experience in domain specific search, search engine evaluation and running evaluation campaigns. She has been coordinating the CLEF-IP evaluation campaign and organising workshops, where specific Information Retrieval methods for the Intellectual Property domain have been assessed. Dr. Piroi is a Lab Chair for CLEF 2021 (Cross-Language Evaluation 
Forum) and has also been on the Organisation Committee of the European Conference for Information Retrieval (ECIR) 2015. Since 2017 she is coordinating the Innovation Training Course "Data Science and Deep Learning" at TU Wien. She has received her PhD degree from the Johannes Kepler University, Austria, where her work concentrated on management and retrieval of mathematical knowledge and automatic theorem provers. She is also a co-founder of Artificial Researcher-IT GmbH.

Dr. Dean Alderucci. Dean Alderucci is the director of research for the Center for Artificial Intelligence and Patent Analysis at Carnegie Mellon University. His research involves extracting knowledge from the text of legal and other documents, and automating complex tasks performed by knowledge-intensive workers such as lawyers, regulators, and medical professionals. He also advises organizations on best practices for implementing machine learning and natural language processing technologies, and on creating AI tools customized to various business areas. Dean speaks frequently on applying AI, especially in patent and legal domains. Most recently he was an organizer for the AI and Patent Data workshop at the 33rd International Conference on Legal Knowledge and Information Systems conference and the Learning Network Architecture During Training workshop at the 35th AAAI Conference on Artificial Intelligence. He is also a Peer Reviewer for the Artificial Intelligence and Law Journal. He was previously COO \& Chief IP counsel for a global financial services firm, and before that chief counsel for a business incubator. He is also a registered patent attorney and a Fellow of the National Academy of Inventors.

\section{1ST EDITION OF THE WORKSHOP AT SEMANTICS CONFERENCE 2019}

The PatentSemTech2019 workshop ${ }^{9}$ was the first edition in a planned series of workshops on patent text mining and semantic technologies. We invited scientific as well as proof of concept contributions that show relevant use cases for patent text mining and analytics. We welcomed researchers to investigate and promote new means for training data generation, e.g. for labelling domain-specific data sets from the Intellectual Property (IP) domain. The articles submitted to the workshop went through a peer-review process where each submission was reviewed by at least three reviewers out of a mixed program committee of academic researchers and experts from the IP domain. Seven papers passed the review process (three long, two short and two demo papers). Three submissions that passed the reviewing process were proposed for publication to the World Patent Information (WPI) journal's virtual special issue on "Patent Text Mining and Semantic Technologies". ${ }^{10}$ For these submissions we only included their (extended) abstracts in the workshop proceedings published at reposiTUm ${ }^{11}$.

In its first year, the workshop was organized as a one day event with a rich program comprising a keynote, paper presentations, demos and a Fishbowl discussion. The keynote was delivered by Mr. A. Trippe, managing director of Patinformatics LLC., a senior, internationally recognised IP expert, and an adjunct Professor of IP

\footnotetext{
${ }^{9} \mathrm{http}: / /$ ifs.tuwien.ac.at/patentsemtech/2019/

${ }^{10} \mathrm{https} / /$ www.journals.elsevier.com/world-patent-information/call-for-papers/textmining-and-semantic-technologies

${ }^{11}$ https://repositum.tuwien.at/handle/20.500.12708/835
}

Management and Markets at Illinois Institute of Technology where he teaches courses on patent analysis and landscapes for strategic decision making. His keynote addressed the importance of patent analytics tools based on semantics and machine learning techniques for the strategic decisions that businesses need to take with respect to their long term R\&D and economic plans.

The participants' feedback was positive with the recommendation to continue the good mix of scientific and practical presentations and the demos. The participating experts expressed that such events are too rare, though highly welcomed by both IP experts and academic researchers.

\section{REFERENCES}

[1] Linda Andersson, Hidir Aras, Florina Mihaela Piroi, and Allan Hanbury (Eds.). 2019. Proceedings of the 1st Workshop on Patent Text Mining and Semantic Technologies (PatentSemTech). Institut für Information Systems Engineering, TU Wien, Austria. https://doi.org/10.34726/pst2019

[2] Linda Andersson, Mihai Lupu, João Palotti, Allan Hanbury, and Andreas Rauber. 2016. When is the Time Ripe for Natural Language Processing for Patent Passage Retrieval?. In Proceedings of the 25th ACM International on Conference on Information and Knowledge Management (Indianapolis, Indiana, USA) (CIKM '16). Association for Computing Machinery, New York, NY, USA, 1453-1462. https://doi.org/10.1145/2983323.2983858

[3] Geert Asche. 2017. "80\% of technical information found only in patents" - Is there proof of this [1]? World Patent Information 48 (2017), 16-28. https://doi.org/10. 1016/j.wpi.2016.11.004

[4] Gaetano Cascini and Davide Russo. 2007. Computer-aided analysis of patents and search for TRIZ contradictions. International fournal of Product Development 4, 1/2 (2007), 52-67. https://ideas.repec.org/a/ids/ijpdev/v4y2007i1-2p52-67.html

[5] Allan Hanbury, Veronika Zenz, and Helmut Berger. 2010. 1st international workshop on advances in patent information retrieval (AsPIRe'10). SIGIR Forum 44, 1 (2010), 19-22. https://doi.org/10.1145/1842890.1842893

[6] Noriko Kando and Mun-Kew Leong. 2000. Workshop on Patent Retrieval (SIGIR 2000 Workshop Report). SIGIR Forum 34, 1 (2000), 28-30. https://doi.org/10.1145/ 373593.373621

[7] Hang Li and Zhengdong Lu. 2016. Deep learning for information retrieval. In Proceedings of the 39th International ACM SIGIR conference on Research and Development in Information Retrieval. ACM, New York, USA, 1203-1206.

[8] Julian Risch, Nicolas Alder, Christoph Hewel, and Ralf Krestel. 2020. PatentMatch: A Dataset for Matching Patent Claims \& Prior Art. CoRR abs/2012.13919 (2020), 5 pages. arXiv:2012.13919 https://arxiv.org/abs/2012.13919

[9] Julian Risch and Ralf Krestel. 2019. Domain-specific word embeddings for patent classification. Data Technol. Appl. 53, 1 (2019), 108-122. https://doi.org/10.1108/ DTA-01-2019-0002 